\title{
Optimizing photodynamic therapy by liposomal formulation of the photosensitizer pyropheophorbide-a methyl ester: In vitro and ex vivo comparative biophysical investigations in a colon carcinoma cell line
}

\author{
Pierre-Henri Guelluy, ${ }^{* a}$ Marie-Pierre Fontaine-Aupart,${ }^{b}$ Angeliki Grammenos, ${ }^{a}$ Sandrine Lécart, ${ }^{c}$ \\ Jacques Piette $^{d}$ and Maryse Hoebeke ${ }^{a}$
}

Received 23rd April 2010, Accepted 24th July 2010

First published as an Advance Article on the web 12th August 2010

DOI: 10.1039/c0pp00100g

Photodynamic therapy (PDT), induced by a photosensitizer (PS) encapsulated in a nanostructure, has emerged as an appropriate treatment to cure a multitude of oncological and non-oncological diseases. Pyropheophorbide-a methyl ester (PPME) is a second-generation PS tested in PDT, and is a potential candidate for future clinical applications. The present study, carried out in a human colon carcinoma cell line (HCT-116), evaluates the improvement resulting from a liposomal formulation of PPME versus free-PPME. Absorption and fluorescence spectroscopies, fluorescence lifetime measurements, subcellular imaging and co-localization analysis have been performed in order to analyze the properties of PPME for each delivery mode. The benefit of drug encapsulation in DMPC-liposomes is clear from our experiments, with a 5-fold higher intracellular drug delivery than that observed with free-PPME at similar concentrations. The reactive oxygen species (ROSs) produced after PPME-mediated photosensitization have been identified and quantified by using electron spin resonance spectroscopy.

Our results demonstrate that PPME-PDT-mediated ROSs are composed of singlet oxygen and a hydroxyl radical. The small amounts of PPME inside mitochondria, as revealed by fluorescence colocalization analysis, could maybe explain the very low apoptotic cell death measured in HCT-116 cells.

\section{Introduction}

Photodynamic therapy (PDT) is a polyvalent medical technique using a photosensitizer (PS) as curative molecule, light radiation from near-UV to near-infrared as an activator, and oxygenated tissues or cells. PDT appears to be not only a powerful technique against several common types of cancer such as skin, ${ }^{1}$ prostate, ${ }^{2}$ ovarian, ${ }^{3}$ breast, ${ }^{4}$ bladder, ${ }^{5}$ and oesophagal cancer, ${ }^{6}$ but also against severe non-oncological diseases like psoriasis, ${ }^{7}$ macular degeneration, ${ }^{8}$ fungal infections, ${ }^{9}$ and dermatoses. ${ }^{10}$ After irradiation under aerobic condition, the drug provokes the direct or indirect production of reactive oxygen species (ROSs) such as singlet oxygen $\left({ }^{1} \mathrm{O}_{2}\right)$, superoxide anion $\left(\mathrm{O}_{2}{ }^{\cdot-}\right)$ and hydroxyl radical $\left(\mathrm{OH}^{*}\right)$, or other radical species responsible for cell death by necrotic and/or apoptotic pathways. ${ }^{11}$

As one of the first generation of photosensitizers, Photofrin ${ }^{\circledR}$ became the most popular, and was the first licensed for clinical use of PDT in 1993 - however, it presents a number of drawbacks. ${ }^{11}$ Among them we can list weak activation above $600 \mathrm{~nm}$ (wavelength range with the deepest penetration power of visible light through tissues), a low selectivity for tumors and prolonged skin photosensitivity. These drawbacks have encouraged the synthesis

${ }^{a}$ Laboratory of Biomedical Spectroscopy, Institute of Physics, B5a, University of Liège, Sart-Tilman, B-4000, Liège, Belgium. E-mail: phguelluy@ ulg.ac.be; Fax: +32 (0)436636 29; Tel: +32 (0)43663631

${ }^{b}$ Institut des Sciences Moleculaires d'Orsay, FRE 3363, University of ParisSud 91405 Orsay, France - CNRS, 91405, Orsay, France

${ }^{\circ} C P B M / L U M A T, F R$ 2764, University of Paris-Sud 91405 Orsay, France ${ }^{d} G I G A-R$ : Virology and Immunology, B34, University of Liège, B-4000, Liège, Belgium of more efficient molecules, much more adapted for clinical applications and patient comfort. Pyropheophorbide-a methyl ester (PPME), a derivative of chlorophyll-a, is one of this new generation of PSs and has been studied in vitro for nearly a decade on cancerous cells such as lung carcinoma NCI-h446, ${ }^{12}$ colon carcinoma HCT-116, ${ }^{13,14}$ prostate adenocarcinoma PC-3M ${ }^{15}$ and nasopharyngeal carcinoma NPC. ${ }^{16}$ Its potential fields of action seem to be numerous and diverse, making this molecule a potent candidate for future human in vivo treatments.

It has been previously established that PPME is a molecule capable of inducing apoptotic and necrotic cell death. ${ }^{13,14}$ The type of cell mortality induced by PPME, correlated with its intracellular localization, has been intensively studied by Matroule ${ }^{13,14}$ and Sun. ${ }^{12}$ These authors found discordance in the localization of PPME within mitochondria in the cell lines investigated, HCT116 and NCI-h446. The localization of a PS inside mitochondria is currently the subject of much study. Indeed, such cellular localization could be the trigger for an apoptotic cell death mechanism, ${ }^{17,18}$ which is always preferred as it does not result in inflammation after treatment. The aggregation state of PS, especially in aqueous solvents, likewise plays a fundamental role in the death process. ${ }^{19}$ For example, the photoactivity of PPME in phosphate buffer is nearly completely abolished because of its hydrophobicity. The aggregated form of PPME in this solvent has two consequences - namely, that the ${ }^{1} \mathrm{O}_{2}$ production disappears completely, and that the production of $\mathrm{OH}^{*}$ is considerably reduced. ${ }^{20}$ To overcome such problems, formulation of waterinsoluble drugs is required, and nanocarriers appear to be elegant and convenient drug delivery systems in this respect. The carriers of PSs must be able to preserve the activity of the entrapped 
PS in its monomeric state once in aqueous solution, to stop damage caused by the bloodstream passage, to release the drug intracellularly and to display high tumor affinity. Liposomal formulations have emerged as an attractive system due to their versatile structure (size, flexibility, unilamellar or multilamellar, lipidic constituent, specific surface functionalization adaptable to various experimental conditions). ${ }^{21-24}$ Liposomes have also a natural affinity for cancer cells because of the presence of an exceptionally high number of lipoproteins in the cytoplasmic membrane of these abnormal cells.

In a first approach to PS vectorization inside a cancer cell line, a classic liposome like dimyristoyl-L- $\alpha$-phosphatidylcholine (DMPC) is a relevant choice for drug delivery. In the present study, we compared the antitumor efficacy of encapsulated PPME in DMPC-liposomes (Lipo-PPME) versus free-PPME (PPME) on a human colon carcinoma cell line, HCT-116. We started this comparative study by an absorption and fluorescence microspectroscopic analysis of the PS properties. Multimodal confocal microscopy (fluorescence intensity, spectra and lifetime) allowed us to determine the mechanism of PPME release by liposomes, the drug's subcellular localization, as well as its intracellular activity. The generation and identification of the ROSs were evaluated by an electron spin resonance (ESR) spin trapping technique, whereas a flow cytometric analysis supplied cellular viability/death and necrotic/apoptotic ratios.

\section{Materials and methods}

\section{Cell culture}

The HCT-116 (human colon carcinoma) cell lines, used at earlypassage (5 to 20), were grown in Dulbecco's Modified Eagle's Medium culture medium (DMEM, Invitrogen), supplemented with $10 \%$ heat-inactivated FBS (Foetal Bovine Serum, Invitrogen), $1 \%$ L-glutamine (Invitrogen) and 1\% mixture of penicillin and streptomycin (Invitrogen) at $37{ }^{\circ} \mathrm{C}$ in a humidified $5 \% \mathrm{CO}_{2}$ incubator.

\section{PPME-liposome preparation}

Stock solution of pyropheophorbide-a methyl ester (PPME, 95\% purity, Sigma Chemical Co., St. Louis, MO, USA) was prepared in ethanol $(400 \mu \mathrm{M})$ and kept in the dark at $-20{ }^{\circ} \mathrm{C}$ for less than 6 months. Unilamellar dimyristoyl-L- $\alpha$-phosphatidylcholine (DMPC, Sigma)-PPME liposomes (Lipo-PPME) were prepared using an extrusion procedure as described previously. ${ }^{25-26}$ In brief, $40 \mathrm{mg}$ of DMPC phospholipids were dissolved in $7 \mathrm{ml}$ chloroform and mixed with $1 \mathrm{ml}$ of PPME before evaporation

Multilamellar vesicles were prepared by mechanical stirring (vortex mixer) of the lipid-PPME film resuspended in $8 \mathrm{ml}$ Phosphate Buffer Saline (PBS, $\mathrm{pH}=7.00$ ) at a temperature above $23.9{ }^{\circ} \mathrm{C}$, phase transition temperature (PTT) of DMPC. To improve incorporation of PPME into liposomes, 5 cycles of freezing-thawing were performed by plunging the solution into liquid nitrogen and then into water at a temperature above the PTT of DMPC. Unilamellar liposomes were formed by extrusion of the suspension through polycarbonate filters $(0.1 \mu \mathrm{m}$ pore size, Nucleopores, Pleasanton, CA) using a commercial extruder apparatus thermostatted at $35^{\circ} \mathrm{C}$. The procedure was repeated
10 times to obtain homogeneously sized liposome dispersion. ${ }^{27} \mathrm{~A}$ similar protocol was used for Lipo-PPME doped with Bodipy FL $\mathrm{C}_{5}(5 \mu \mathrm{M}$, Invitrogen). The Bodipy was mixed with DMPC and PPME before evaporation. The stability of both types of liposomes was confirmed by absorption and fluorescence spectroscopic measurements.

\section{Exposure of HCT-116 cells to drug photosensitization}

For photosensitization experiments (absorption, fluorescence, flow cytometry, ESR), before irradiation, HCT-116 cells were incubated with PPME (1 to $5 \mu \mathrm{M})$ or Lipo-PPME $(0.1$ to $5 \mu \mathrm{M})$ for $20 \mathrm{~h}$. It must be noted that drug concentrations higher than $5 \mu \mathrm{M}$ are deleterious to cell survival due to ethanol (EtOH) contained in the stock solution. Cells were then washed once with PBS, incubated 2 min with trypsin (Invitrogen), the latter being inactivated by DMEM containing $10 \%$ of FBS. Centrifugation at $1500 \mathrm{rpm}$ for $5 \mathrm{~min}$ was carried out in order to eliminate the supernatant before replacing the cellular pellet in DMEM without FBS. In the presence or absence (control experiments) of PS, HCT-116 cells were then irradiated with a $\mathrm{Hg}$-vapor lamp (fluence rate of $245 \mathrm{~W} / \mathrm{m}^{2}$ for a period of $4.5 \mathrm{~min}$, corresponding to $66 \mathrm{~kJ} / \mathrm{m}^{2}$ ) equipped with a cut-off filter eliminating wavelengths under $600 \mathrm{~nm}$.

\section{Flow cytometry experiments - cell mortality}

After irradiation as described above, HCT-116 cells were put back in culture for $24 \mathrm{~h}$ at $37^{\circ} \mathrm{C}$ in DMEM supplemented with $\% \mathrm{FCS}$. HCT-116 cells were then once again washed with PBS, trypsinized, centrifuged and resuspended in the HEPES buffer. Cells were then stained as described by the manufacturer (Annexin-V-Fluos kit from ROCHE). Briefly, Annexin-V-fluorescein (Ann) and propidium iodide (PI) are added and incubated in the dark for 15 min before analysis with a FacsCanto II flow cytometer (BD Biosciences). We used a standard $515 \mathrm{~nm}$ bandpass filter $(515 \pm 21 \mathrm{~nm})$ for Ann-fluorescence detection and a $585 \mathrm{~nm}$ bandpass filter $(585 \pm 15 \mathrm{~nm})$ to detect only the fluorescence of PI without interference with PPME-fluorescence (ranging from 650$750 \mathrm{~nm}$ ). The percentage of living cells corresponds to a negative fluorescence of Ann $(\operatorname{Ann}(-))$ and a negative fluorescence of PI (PI(-)), necrotic cells to Ann(-) and PI(+), or Ann(+) and PI(+), and apoptotic cells to Ann(+) and PI(-).

\section{Confocal laser scanning microscopy - co-localization experiments}

Fluorescence images and co-localization experiments were performed with a LEICA TCS SP2 (University of Liège, Giga, Liège, Belgium) or a SP5 AOBS (Centre de Photonique Biomédicale, Orsay, France) confocal microscope. Cells were observed using a $63 \times-1.4$ numerical aperture (NA) oil immersion objective. The resolution of the confocal images was $512 \times 512$ pixels (corresponding to an area $246.03 \times 246.03 \mu \mathrm{m}^{2}$ ), recorded on 12 bits with a zoom value of 1 . All confocal laser scanning microscopy experiments were performed 4 times with similar results. A helium-neon laser with maximum wavelength at $633 \mathrm{~nm}$ was used as the excitation source for PPME and Lipo-PPME; the PS fluorescence was collected in the spectral range $650-725 \mathrm{~nm}$. Bodipy fluorescence was recorded in the spectral range 500 $600 \mathrm{~nm}$ with a $\lambda_{\text {exc }}$ of $488 \mathrm{~nm}$. 
For co-localization experiments, we used a $6.5 \times$ zoom in order to have the most appropriate lateral resolution $\left(d_{x y}=71.52 \mathrm{~nm}\right)$ according to the following equation:

$$
d_{x y}=\frac{0.46 \times \lambda_{\mathrm{em}}}{\mathrm{NA} \times 2.3}
$$

where $\lambda_{\mathrm{em}}$ is the shortest emission wavelength of each experiment. The factor 2.3 is the direct consequence of the Shannon-Nyquist theorem and 0.46 is a theoretical factor. The best axial resolution $\left(d_{z}=160.25 \mathrm{~nm}\right)$ was similarly determined by the following equation:

$$
d_{z}=\frac{1.4 \times \lambda_{\mathrm{em}}}{\mathrm{NA}^{2} \times 2.3}
$$

Colocalization analysis was performed using JACoP v2.0, a plug-in running under ImageJ software, developed by Bolte and Cordelières. ${ }^{28}$ The statistical method provides the Pearson coefficient ( $P$-value) which varies between -1 (anti-co-localization) and +1 (total co-localization) and reflects an unbiased co-localization of two fluorescent probes as tested by Costes' method. ${ }^{29}$ In the present work, a $P$-value $>+0.15$ was considered statistically significant for co-localization of two probes.

Fluorescence lifetime imaging measurements (FLIMs) were performed under two-photon excitation at a fixed wavelength of $800 \mathrm{~nm}$. For this purpose, the SP5 confocal microscope was coupled to a titania:sapphire laser providing $90 \mathrm{MHz}$ modelocked pulses (Chameleon-XR, Coherent). The fluorescence signal was collected by a time-correlated single-photon counting module SymphoTime 300 (PicoQuant) equipped with an avalanche photodiode.

Three days prior to the experiments, HCT-116 cells were cultivated in a Labtek 2-well slide chamber as described previously and incubated with PPME or Lipo-PPME for the last $20 \mathrm{~h}$. Before experiments, the supernatant containing PPME or Lipo-PPME was removed and replaced by fresh DMEM.

Mitochondria were stained by incubating them for $45 \mathrm{~min}$ in DMEM-treated and control cells with $200 \mathrm{nM}$ of Mitotracker Green (Invitrogen) $\left(\lambda_{\mathrm{exc}}=488 \mathrm{~nm}\right.$; fluorescence emission 500$610 \mathrm{~nm}$ ) before the confocal experiments. It must be noted that no fluorescence of DMEM was observed in this spectral range of acquisition.

\section{ESR experiments}

The ESR measurements were performed with an EPR 300E Spectrometer (Bruker) operating at the X-band $(9.56 \mathrm{GHz})$. Instrumental settings were: modulation frequency $=100.00 \mathrm{kHz}$, amplitude modulation $=2.42 \mathrm{G}$, receiver gain $=1 \times 10^{5}$, conversion time $=81.92 \mathrm{~ms}$, time constant $=40.96 \mathrm{~ms}$, resulting sweep time $=$ $83.89 \mathrm{~s}$, number of scans $=6$, microwave frequency $=9.56 \mathrm{GHz}$, microwave power $=20.2 \mathrm{~mW}$, width sweep $=100 \mathrm{G}$ and centre field $=3480 \mathrm{G}$.

For these ESR experiments, control and treated cells were resuspended in Hawk's balanced saline solution, supplemented with $100 \mathrm{mM}$ of the spin trap $\alpha$-(4-pyridyl-1-oxide)- $N$-tert-butylnitrone (POBN, Sigma) and 2\% EtOH. Lastly, ROS quenchers (1,4diazabicyclo[2.2.2]octane (DABCO, Sigma), superoxide dismutase (SOD, bovine erythrocytes, Sigma), catalase (CAT, from bovine liver, Sigma)) or chelator (Desferrioxamine (DES, Sigma)) were added to obtain a final volume of $500 \mu \mathrm{l}$. After $10 \mathrm{~min}$ of incubation with POBN-EtOH and final quenching, cell suspensions were irradiated as described previously $\left(66 \mathrm{~kJ} / \mathrm{m}^{2}\right)$ and then immediately transferred and analysed by ESR in a quartz flat cell at room temperature.

The choice of the spin trap POBN is motivated by its ability to localize inside cells, ${ }^{30}$ in contrast to DMPO usually used for ROS detection, which did not penetrate inside this particular cell line. Nevertheless, the spin adduct created by POBN and superoxide anion is unstable ${ }^{31}$ and the lifetime of the spin adduct POBN-OH${ }^{*}$ is too short ( $23 \mathrm{~s}$ ) to be detected by the ESR set-up. To counteract this problem, $\mathrm{EtOH}$ ( $2 \%$, a non-toxic concentration for cells) was added in the sample before light illumination in order to scavenge $\mathrm{OH}^{\cdot}$ precursor (rate constant of the reaction $1.9 \times 10^{9} \mathrm{M}^{-1} \mathrm{~s}^{-1}$ ). The ethoxy radical $\left({ }^{\circ} \mathrm{CH}(\mathrm{OH}) \mathrm{CH}_{3}\right)$ formed afterwards ${ }^{32}$ can be trapped by POBN to form POBN-ethoxy adducts (rate constant $=$ $3 \times 10^{7} \mathrm{M}^{-1} \mathrm{~s}^{-1}$ ), which appear as a triple double-lined spectrum (hyperfine splitting: $a^{\mathrm{N}}=15.7 \mathrm{G} ; a^{\mathrm{H}}=2.7 \mathrm{G}$ ).

\section{Absorption and fluorescence spectroscopy}

The absorption and fluorescence spectra were recorded using a Kontron double-beam spectrophotometer (Uvikon 941) and a SLM-Aminco 500 spectrofluorimeter (SLM instruments), respectively. The samples were placed in quartz (Suprasil) cells $(0.4 \mathrm{~cm}$ width $\times 1 \mathrm{~cm}$ path length, Hellma, Belgium).

The fluorescence measurements were carried out at a fixed excitation wavelength of $417 \mathrm{~nm}$. For cellular determination of fluorescence, HCT-116 cells were isolated from supernatant in order to determine separately the fluorescence of intracellular and extracellular PPME. The aggregation state of PPME in aqueous media rendered possible this comparison of extra and intracellular fluorescence when the drug was encapsulated in liposomes. We decided not to explore absorption spectra including cells; indeed, the significant scattering induced by cells produced unreliable results. The determination of saturation drug penetration has not been achieved for free-PPME; the cytotoxicity of EtOH as PPME solvent eliminates the possibility of using concentrations higher than $5 \mu \mathrm{M}$ (initial PPME stock solution of $400 \mu \mathrm{M}$ ).

\section{Results}

\section{Absorption and fluorescence spectroscopy}

We have previously reported the absorption and fluorescence spectra of PPME dissolved in ethanol and in phosphate-buffered saline $(\mathrm{PBS})^{20}$ when the molecule is in its free form or incorporated in a liposome.

Free-PPME in PBS aggregates, reducing strongly its photoactivity, whereas Lipo-PPME retains high photoactivity in ethanol and PBS. ${ }^{20}$ Thus, before any cell experiments, we controlled the ability of PPME and Lipo-PPME to aggregate in the cell culture medium DMEM (Fig. 1(a) and Fig. 1(b)). The absorption and fluorescence spectra after $20 \mathrm{~h}$ of incubation (in order to recreate the conditions of incubation in the cell medium) are identical to those previously reported in PBS. ${ }^{20}$ They show characteristics of PPME in its monomeric form inside liposomes and of aggregates in DMEM, 
Table 1 Spectral photostability of PPME and Lipo-PPME in DMEM culture medium after exposure to progressively higher light doses

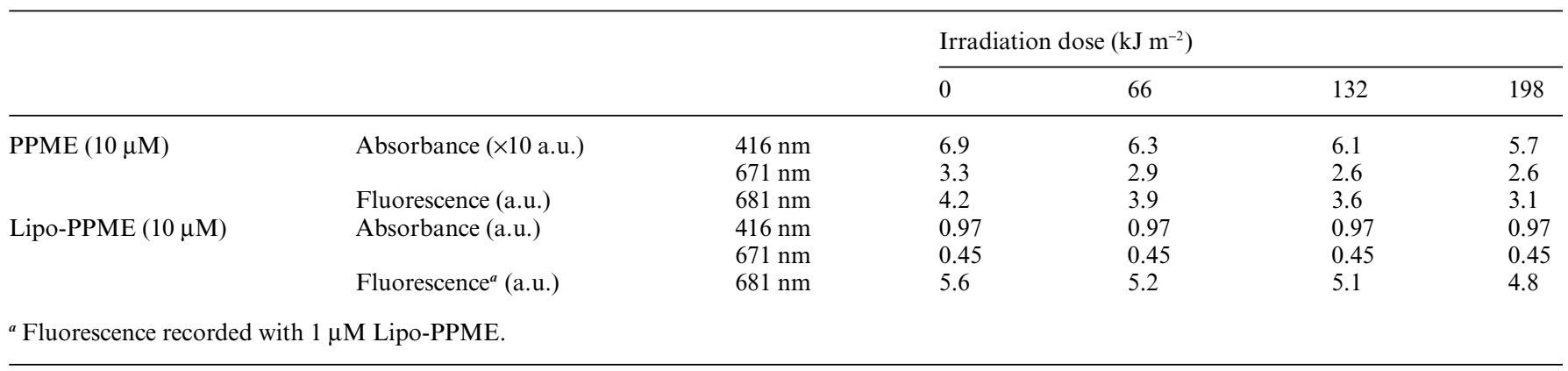

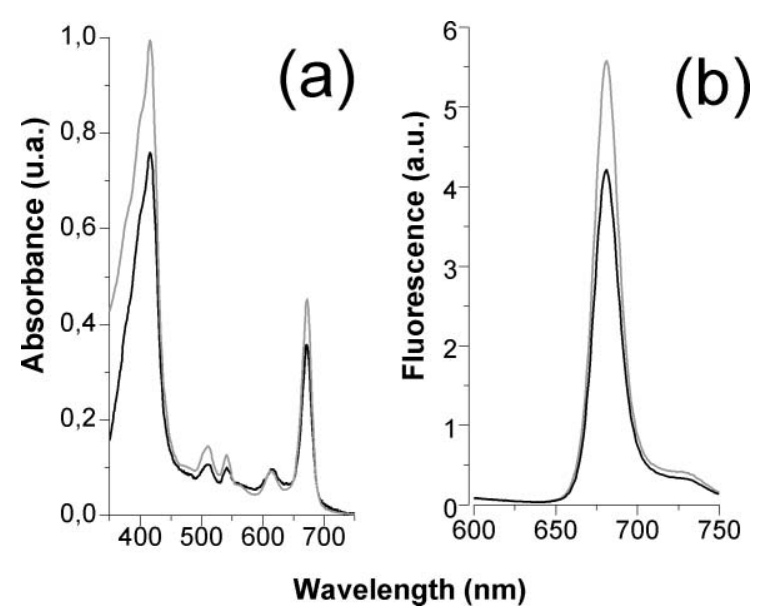

Fig. 1 (a) Absorption spectrum of $10 \mu \mathrm{M}$ PPME (black trace) (the absorbance has been multiplied by 10 for a better interpretation of results) and $10 \mu \mathrm{M}$ Lipo-PPME (grey trace), both incubated $20 \mathrm{~h}$ in DMEM. (b) Fluorescence spectrum $\left(\lambda_{\text {exc }}=416 \mathrm{~nm}\right)$ of $10 \mu \mathrm{M}$ PPME (black trace) and $1 \mu \mathrm{M}$ Lipo-PPME (grey trace) under the same incubation conditions.

as revealed by the significant decrease of both absorption and fluorescence spectra intensity. ${ }^{20}$

The photostability of PPME and Lipo-PPME (Table 1) was also evaluated from absorption and fluorescence spectra. Several irradiation cycles were applied, using typical PDT light doses ranging from $66 \mathrm{~kJ} \mathrm{~m}^{-2}$ to $198 \mathrm{~kJ} \mathrm{~m}^{-2}$. $^{12}$ After 3 cycles of irradiation, Lipo-PPME was found to be light-stable while for free-PPME maximum intensity decreases of $15 \%$ and $24 \%$ for absorbance and fluorescence were observed respectively (Table 1). Under the experimental conditions of this study $\left(66 \mathrm{~kJ} \mathrm{~m}^{-2}\right)$ both PPME and Lipo-PPME are photostable.

Quantification of intracellular PPME incorporation was also determined by fluorescence spectroscopy. After incubation of HCT-116 cells with $1 \mu \mathrm{M}$ Lipo-PPME for $20 \mathrm{~h}$, supernatant was separated from treated cells. The spectra in Fig. 2(a) show that Lipo-PPME fluorescence intensity corresponds to a perfect subdivision of the extra and intracellular signals; only onethird of the monomeric drug was able to penetrate the cells. Experiments were further performed for various Lipo-PPME concentrations, and the resulting fluorescence spectra of treated cells are represented in Fig. 2(b). A saturation of drug uptake was reached for initial Lipo-PPME concentrations $\geq 1 \mu \mathrm{M}$, taking into account experimental precision.

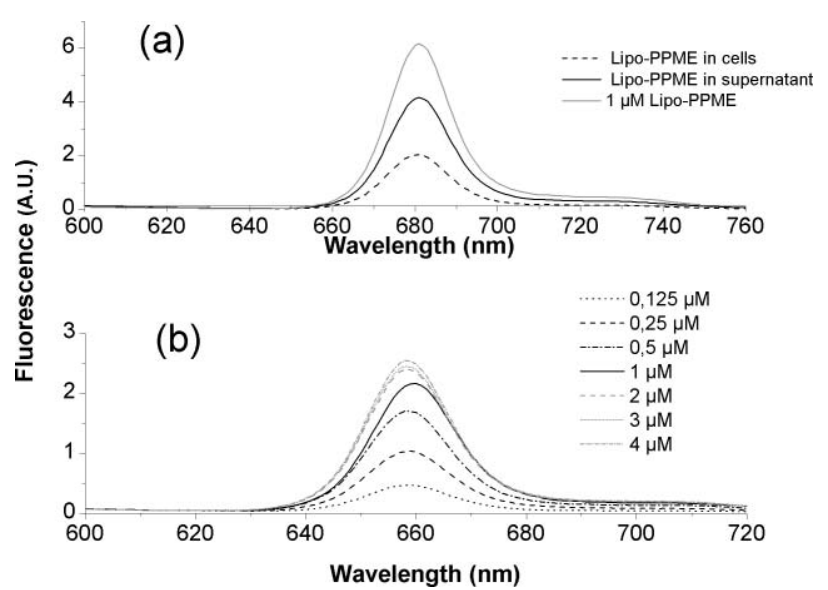

Fig. 2 (a) Fluorescence spectrum of Lipo-PPME in presence of HCT-116 cells (excitation of $417 \mathrm{~nm}$ ). The grey trace corresponds to the fluorescence of $1 \mu \mathrm{M}$ Lipo-PPME in DMEM after $20 \mathrm{~h}$ of incubation. The black trace is the remaining fluorescence of Lipo-PPME in supernatant medium after $20 \mathrm{~h}$ of incubation. The black dotted trace is the fluorescence of PPME inside HCT-116 cells after $20 \mathrm{~h}$ of incubation with $1 \mu \mathrm{M}$ Lipo-PPME. (b) Fluorescence spectra of PPME inside HCT-116 cells after incubation (20 h) with various concentrations of Lipo-PPME.

\section{Cellular uptake of PPME}

Confocal imaging was used to compare the PPME penetration rate inside HCT-116 cells resulting from incubation with free or DMPC-encapsulated molecules. Fluorescence occurs mainly in internal compartments of the cell cytoplasm other than the nucleus, as highlighted in Fig. 3a-d. In order to determine the cellular uptake in function of the drug delivery system, a statistical analysis of intracellular PPME fluorescence was carried out with two different concentrations (1 and $5 \mu \mathrm{M}$ ) of PPME (Fig. 3a and Fig. 3b) and Lipo-PPME (Fig. 3c and Fig. 3d). The upper fluorescence intensity profiles (FIP) were achieved for $5 \mu \mathrm{M}$ PPME and for $1 \mu \mathrm{M}$ Lipo-PPME, which confirms the result reported above by microspectrofluorimetry. It is also relevant to notice that Fig. 3b, Fig. 3c and Fig. 3d have the same FIP that corresponds to the upper drug uptake by HCT-116 cells. This means that, under such incubation conditions, no more monomeric PPME is able to penetrate intracellularly. The use of a liposome thus has the possibility to improve by a factor of 5 the PPME cellular uptake (Fig. $3 \mathrm{~b}$ and Fig. 3d).

Furthermore, these results demonstrated that incubation with concentrations higher than $5 \mu \mathrm{M}$ PPME or $1 \mu \mathrm{M}$ Lipo-PPME 


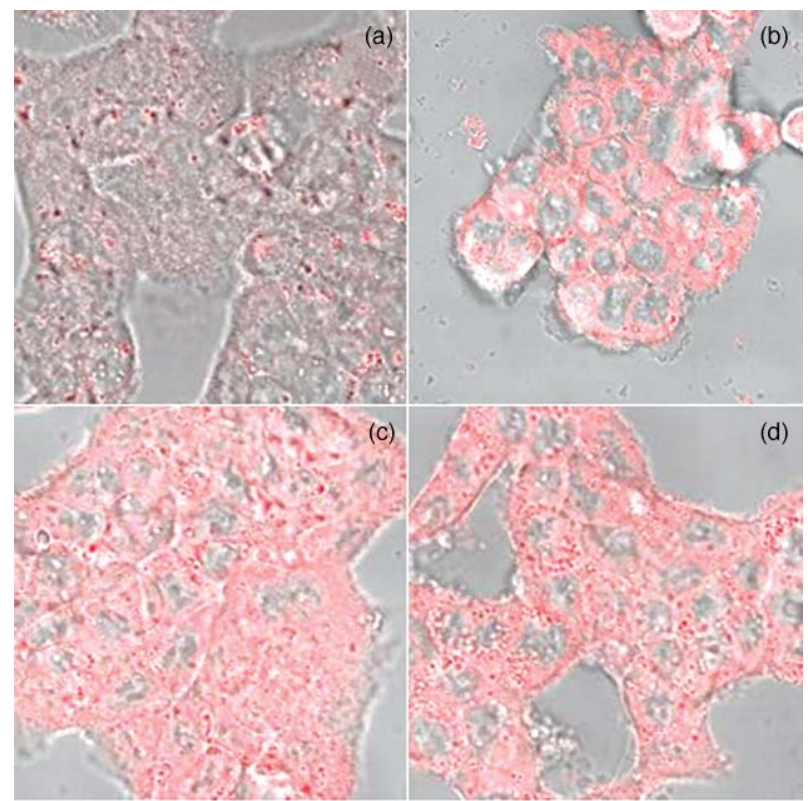

Fig. 3 Confocal laser scanning microscopy of PPME inside HCT-116 cells. (a): Incubation with $1 \mu \mathrm{M}$ PPME. (b): Incubation with $5 \mu \mathrm{M}$ PPME. (c): Incubation with $1 \mu \mathrm{M}$ Lipo-PPME. (d): Incubation with $5 \mu \mathrm{M}$ Lipo-PPME.

respectively definitely appears not to be advantageous. Timeresolved fluorescence measurements were also performed in several points inside cells after incubation with PPME or LipoPPME. Whatever the deliverance mode, a nanosecond fluorescence lifetime $(6.0 \pm 0.5 \mathrm{~ns})$ was measured over the whole cell. This value is similar to the lifetime of PPME in ethanol and Lipo-PPME in buffer solution, as previously reported, ${ }^{20}$ and corresponds to the monomeric form of the PS.

\section{Mechanism of drug release from Lipo-PPME}

Confocal microscopy also allowed us to determine if the liposomes were taken up by cells by endocytosis mechanisms, fusion or other processes. The use of Lipo-PPME doped with Bodipy, a fluorescent probe strongly trapped within DMPC-liposomes, rendered possible direct visualization of these nanostructures. A fluorescence image of HCT-116 cells incubated in a culture medium containing doped liposomes, is given in Fig. 4(a). The distribution of Bodipy fluorescence (in green) proves the presence of Lipo-PPME all around the HCT-116 cells but not inside, whereas PPME fluorescence distribution (in red) shows the presence of this dye both extra- and intracellularly. After removal of incubation supernatant, the only fluorescence remaining is the one of PPME, localized only intracellularly (Fig. 4(b)). These results reveal that liposomes do not enter the cells by internalization or fusion mechanisms. PPME is thus released by liposomes at the level of the plasma membrane by intermembrane contact. No fixation of the nanostructure to the cells has been observed so far (Fig. 4(b)).

\section{Co-localization of PPME with mitochondria}

The subcellular localization of PPME $(2 \mu \mathrm{M})$ was first studied in the NCI-h446 cell line. ${ }^{12}$ It was found that PPME is present inside every organelle (endoplasmic reticulum, Golgi, lysosomes and

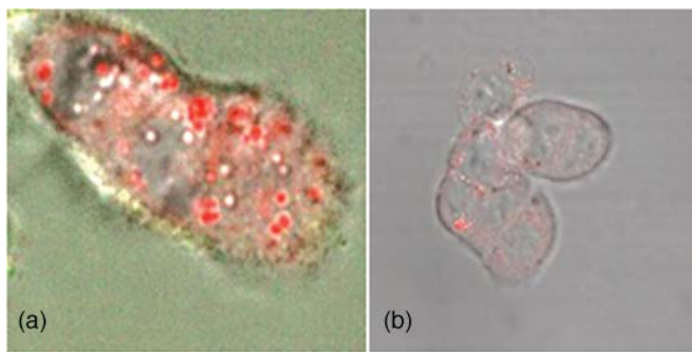

Fig. 4 Confocal laser scanning microscopy of HCT-116 cells incubated with Lipo-PPME doped with Bodipy (fluorescence of PPME in red; fluorescence of Bodipy in green). (a): In the presence of incubation supernatant. (b): In the absence of incubation supernatant.

mitochondria). By contrast, Matroule et al. ${ }^{14}$ have reported that $0.25 \mu \mathrm{M}$ PPME does not localize in the HCT-116 mitochondria. We have re-examined the co-localization of PPME with HCT-116 mitochondria with a larger range of PPME concentrations; the drug being administrated as a free molecule or encapsulated in liposomes.

Fig. 5(a) and Fig. 5(b) show that in some cell regions, PPME and Mitotracker fluorescences overlap (yellow points), revealing mitochondrial localization of PPME.
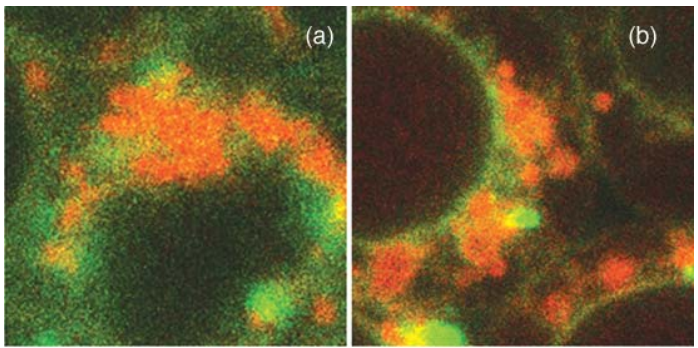

Fig. 5 Confocal laser scanning microscopy of HCT-116 cells incubated with PPME or Lipo-PPME combined with Mitotracker (green). (a): $5 \mu \mathrm{M}$ PPME (red). (b): $1 \mu \mathrm{M}$ Lipo-PPME (red).

In order to quantify this co-localization, we used a statistical method based on evaluation of the Pearson coefficient (see Materials and methods). As we can see in Fig. 6, the values of

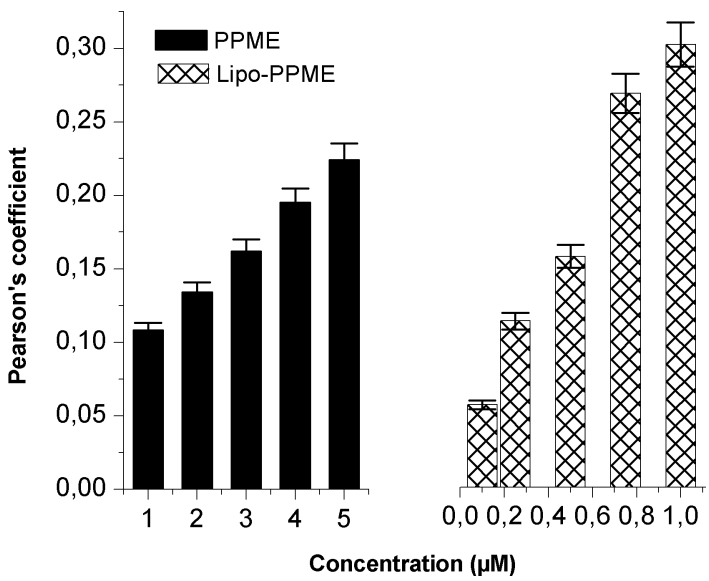

Fig. 6 Mitochondrial PPME Pearson coefficient of co-localization versus concentration of PPME (black bars) or Lipo-PPME (hatched bars) used for incubation with HCT-116 cells. 
the Pearson coefficient $(P)$, which reflects directly the presence of PPME inside mitochondria, increase with the concentration of PPME used during incubation. Furthermore, the results confirm the improvement of cell uptake when PPME is encapsulated in the liposome. Indeed, for an identical PPME concentration of $1 \mu \mathrm{M}$, PC takes a value of 0.1 (no co-localization) for PPME and 0.3 (significant co-localization) for Lipo-PPME. The generally better internalization of PPME induced by liposome is thus also verified at the level of mitochondria. The co-localization of PPME with mitochondria can thus be confirmed for concentrations of freePPME equal to or higher than $4 \mu \mathrm{M}$. With Lipo-PPME, colocalization was already confirmed at a concentration of $0.75 \mu \mathrm{M}$.

\section{ROS production by PDT}

After irradiation of cells incubated with PPME or Lipo-PPME, the characterization of ROS generation was performed by ESR spin trapping using POBN combined with ethanol (2\%). Every spectrum appears as a triple double-lined spectrum, revealing the presence of POBN-ethoxy adducts (see Materials and methods). As appears in Fig. 7A, this adduct is not present without light

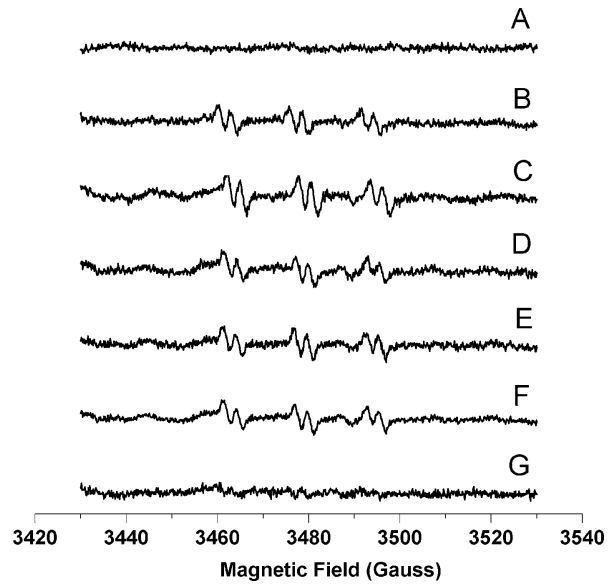

Fig. 7 ESR spectra of HCT-116 cells after $20 \mathrm{~h}$ of drug incubation, combined with POBN $(50 \mu \mathrm{M})$ and EtOH $(2 \%)$ (Spectrum A: without irradiation, spectra B-G: after $66 \mathrm{~kJ} \mathrm{~m}^{-2}$ irradiation). A: $5 \mu \mathrm{M}$ PPME, no light, B: $5 \mu \mathrm{M}$ PPME, C: $1 \mu \mathrm{M}$ Lipo-PPME, D: $1 \mu \mathrm{M}$ Lipo-PPME with CAT $\left(1880 \mathrm{U} \mathrm{ml}^{-1}\right)$, E: $1 \mu \mathrm{M}$ Lipo-PPME with SOD $\left(200 \mathrm{U} \mathrm{ml}^{-1}\right), \mathrm{F}: 1 \mu \mathrm{M}$ Lipo-PPME with DES $(20 \mathrm{mM})$, G: $1 \mu \mathrm{M}$ Lipo-PPME with DABCO $(20 \mathrm{mM})$.

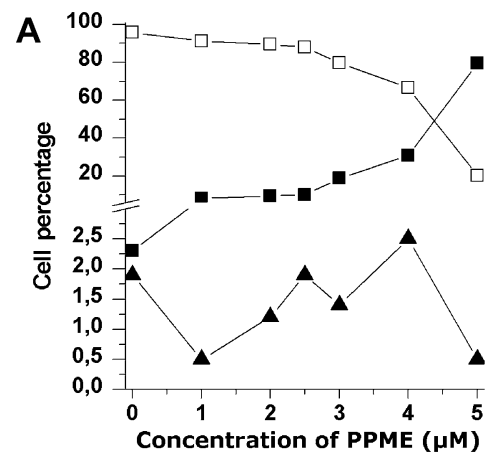

irradiation, proving the inactivity of PPME in the dark. The comparison of ESR spectra obtained with $5 \mu \mathrm{M}$ PPME and $1 \mu \mathrm{M}$ Lipo-PPME (Fig. 7B and Fig. 7C) reveals the great similarity of the signal intensity. Above a concentration of $1 \mu \mathrm{M}$ Lipo-PPME, the intensity of spectrum remains unchanged (data not shown).

A signal intensity reduction of $30 \%$ was observed in the presence of CAT (Fig. 7D), which degrades hydrogen peroxide $\left(\mathrm{H}_{2} \mathrm{O}_{2}\right)$. A similar decrease of $30 \%$ was measured upon addition of SOD (Fig. 7E), which dismutates superoxide anion $\left(\mathrm{O}_{2}^{-}\right)$or with addition of DES (Fig. 7F), an iron(III) chelator. Addition of DABCO (Fig. 7G), a specific quencher of ${ }^{1} \mathrm{O}_{2}$, reduced $70 \%$ of the POBN-ethoxy adduct signal intensity.

These competition experiments clearly reveal that ${ }^{1} \mathrm{O}_{2}$ is the major ROS detected by the POBN-EtOH spin trap after activation of intracellular PPME. Others types of $\operatorname{ROS}\left(\mathrm{H}_{2} \mathrm{O}_{2}, \mathrm{O}_{2}{ }^{--}, \mathrm{OH}^{*}\right)$ represent only $30 \%$ of the ROS detection. It is also relevant to notice that the use of POBN-EtOH combination as a spin trap allows indirect detection of ${ }^{1} \mathrm{O}_{2}$.

\section{Induction of apoptosis and necrosis}

Analysis of the percentage of necrosis/apoptosis following treatment of HCT-116 cells was also performed with either PPME or Lipo-PPME (24 h after irradiation). Flow cytometry analysis was performed by following the intensity of PI/Ann labeling (see Materials and methods).

We found, as shown in Fig. 8A and Fig. 8B, a propensity for necrotic cell death, a percentage that varies from $8 \%(1 \mu \mathrm{M})$ to $79 \%(5 \mu \mathrm{M})$ for PPME, and from $10 \%(0.25 \mu \mathrm{M})$ to $68 \%(1 \mu \mathrm{M})$ for Lipo-PPME. Given that the percentage of apoptotic cells is always between 1 and $3 \%$, we can affirm that necrosis is responsible for HCT-116 cell death. We can also verify the non-toxicity of unactivated PPME towards HCT-116 cells: $5 \mu \mathrm{M}$ of PPME and $1 \mu \mathrm{M}$ Lipo-PPME created respectively a mortality of 5 and $4 \%$ in the absence of any light exposure.

\section{Discussion}

Second-generation PSs, while free from the majority of the drawbacks characterising the first generation, ${ }^{33}$ can still be highly optimized by liposome encapsulation. This modality of cell internalization, created about 40 years ago to localize active drugs in subcellular organelles, is always an active field of research and is especially adapted to hydrophobic molecules such as PPME.

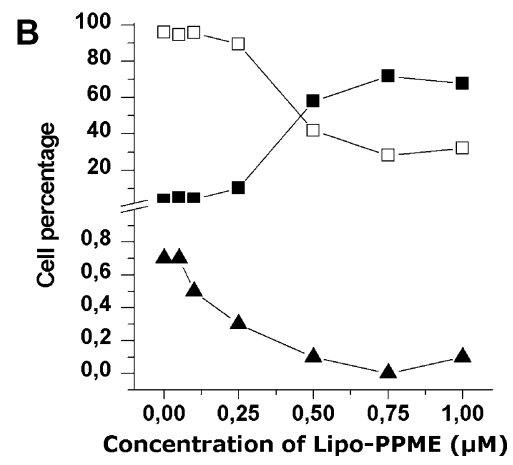

Fig. 8 Percentage of living ( $\square$ ), necrotic PPME. B: Incubation with Lipo-PPME. 
This study has shown that PPME molecules, when embedded inside such nanostructures, are more photostable by comparison with the free-PPME solution. A second advantage linked to the nanocarriers is the ability of these lipidic structures to improve the delivery of PPME inside cells. Indeed, our experiments have proved that whatever the formulation of PPME is, this molecule penetrates inside the cells. What is more noticeable is that a five-fold lower extracellular concentration of LipoPPME is required, by comparison with free-PPME, to obtain the same subcellular localization, the same ROS generation and therefore the same cellular mortality. The drug leakage realized by liposomes is traditionally carried out by phagocytosis or endocytic pathways, this internalization being dependent on the nature of the phospholipids used and the type of target cells. ${ }^{34}$ In the particular situation of combining HCT-116 cell line and DMPC-liposomes (90 $\mathrm{nm}$ in diameter), our results have proven that these liposomes do not enter the cells but directly release PPME at the level of the plasma membrane. The internalization of PPME thus occurs by diffusion, as expected for free-PPME. The fact that liposomes deliver the drug, which is in a monomeric state and directly released to the plasma membrane, has the consequence a significant increase of intracellular concentration of PPME. Our results also enabled us to be certain that, once inside the cells, the drug has the same biochemical and biophysical properties as in solution. No chemical modification or change of the molecular behaviour was observed for PPME introduced freely or by liposomes. This assertion is completely validated by an identical fluorescence lifetime and a similar fluorescence spectrum in both cases. Fluorescence spectroscopy has also shown a monomeric drug saturation level. Indeed, HCT-116 cells have only the ability to internalize a limited amount of PPME, which stays in monomeric state once localized intracellularly. Because aggregated PPME loses its ability to emit fluorescence and to create ROSs after excitation, ${ }^{20}$ this type of molecular structure is not only undetectable by fluorescence techniques but also completely inactive in PDT. The maximum intracellular PPME concentration observed with encapsulated PPME was reached for concentrations between 1 and $2 \mu \mathrm{M}$. Higher concentrations of Lipo-PPME would thus be unnecessary for any treatment using this cellular type; the mortality rates remain approximately the same from 2 to $5 \mu \mathrm{M}$ Lipo-PPME ( $66 \mathrm{~kJ} \mathrm{~m}^{-2}$, data not shown).

The effectiveness of PPME depends on its state of aggregation, but also on its intracellular distribution. The presence of a photosensitizer inside specific organelles can lead to cellular death by necrosis and/or apoptosis. Recently, the subcellular location of PPME (notably inside mitochondria) was still a matter of controversy. ${ }^{14}$ Mitochondrial absorption of PPME could result in apoptosis after production of ROSs in situ. This ROS production would be able to trigger caspase activation, one of the crucial processes of the apoptosis mechanism. ${ }^{35}$ In this study, PPME was found to localize in the endothelial reticulum, the Golgi apparatus and the lysosomes (data not shown), as reported by Matroule et $a{ }^{14}$ on the same HCT-116 cell line and by Sun et al. on NCI-h446. ${ }^{12}$ We also observed that PPME localized inside the mitochondria of HCT-116 cells, as observed for NCI-h446 cells. This discrepancy in subcellular localization with the results of Matroule et al. could be due to the significant difference in extracellular drug concentration used in our experiments and in the co-localization experiments of Matroule $(5 \mu \mathrm{M}$ versus $0.25 \mu \mathrm{M})$.
Indeed, as reported in Table 1, the Pearson coefficients that enabled us to ascertain co-localization (factor $>0.3$ ) correspond to $5 \mu \mathrm{M}$ and $1 \mu \mathrm{M}$ extracellular concentration for PPME and Lipo-PPME respectively. At low extracellular PPME concentrations (lower than $5 \mu \mathrm{M}$ ), the Pearson coefficient is too small to determine the presence of this PS in mitochondria. However, our experiments showed that for every concentration of PPME, even the saturation concentration, the inner mitochondrial PPME concentration remained quite limited (the Pearson coefficients reach a maximal value of 0.3). These low levels of PPME inside mitochondria could, however, be sufficient to explain the caspase activation observed by Matroule. This author had suggested the possible intervention of nomad ROSs produced in organelles other than mitochondria (endoplasmic reticulum and Golgi apparatus) that control caspase activation. This explanation has been ruled out by the simple fact that our co-localization experiments have revealed PPME accumulation inside mitochondria. The activation of caspases is thus probably the direct consequence of ROS production induced by mitochondrial PPME after irradiation. Even if we are now certain that PPME is capable of diffusing inside mitochondria, this organelle is likely not a primary target but an organelle in which PPME will localize when all the cellular sites are filled.

After clarification of the intracellular PPME distribution, its photodynamic activity was examined through its ability to cause cell death. Before any analysis of the consequence of irradiation, we verified and confirmed the non-toxicity of PPME in the dark by flow cytometry measurements and ESR experiments. By flow cytometry measurements, PPME was found to induce both apoptotic and necrotic cell death in a time- and concentrationdependent manner. With a concentration of $5 \mu \mathrm{M}$ PPME, $24 \mathrm{~h}$ after irradiation, necrosis is the major death process $(79 \%)$, compared to apoptosis (3\%). The cellular aggression caused by PDT being ROS-dependent, we used ESR to qualify the type of ROS involved in the cell death process. The diagram below presents the de-excitation of activated-PPME (PPME*) that triggers the production of ROSs inside cells. This de-excitation can occurs by a Type I mechanism that corresponds to a transfer of charge from PPME* leading to the creation of $\mathrm{O}_{2}{ }^{--}$. PPME* can also pass its excess energy to molecular oxygen, in a reaction known as a Type II mechanism, leading this time to the creation of ${ }^{1} \mathrm{O}_{2}$.

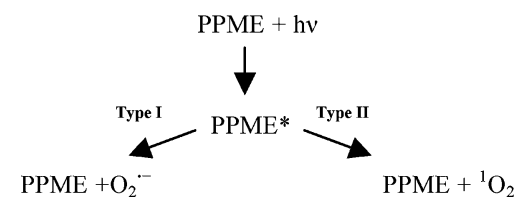

Once $\mathrm{O}_{2}{ }^{--}$is created by Type I mechanism, $\mathrm{OH}^{-}$is produced by the Haber-Weiss (HW) mechanism:

$$
\begin{gathered}
2 \mathrm{O}_{2}^{\cdot-}+2 \mathrm{H}^{+} \rightarrow \mathrm{H}_{2} \mathrm{O}_{2}+\mathrm{O}_{2} \\
\mathrm{Fe}^{3+}+\mathrm{O}_{2}^{\cdot-} \rightarrow \mathrm{Fe}^{2+}+\mathrm{O}_{2} \\
\mathrm{H}_{2} \mathrm{O}_{2}+\mathrm{Fe}^{2+} \rightarrow \mathrm{Fe}^{3+}+\mathrm{OH}^{-}+\mathrm{OH}^{\cdot}
\end{gathered}
$$

Interaction between $\mathrm{OH}^{*}, \mathrm{EtOH}$ and $\mathrm{POBN}$ ends in the formation of the POBN-ethoxy adduct that we observed by ESR. For each quencher used to block one-by-one all the stages of 
the HW mechanism (SOD, DES and CAT respectively for $I_{a}$, $I_{b}$ and $I_{c}$ ), a decrease of the signal intensity of nearly $30 \%$ was measured. The extinction of Type II ROS production, achieved by DABCO, diminished the ESR signal by $\sim 70 \%$. To explain this last observation, we postulated the fact that ${ }^{1} \mathrm{O}_{2}$ is able to react with POBN by forming a complex:

$$
\mathrm{POBN}+{ }^{1} \mathrm{O}_{2} \rightarrow\left[\mathrm{POBN}-{ }^{1} \mathrm{O}_{2}\right]
$$

This complex is followed by a decay by which an adduct and an extra free $\mathrm{OH}^{*}$ are generated:

$$
\left[\mathrm{POBN}-{ }^{1} \mathrm{O}_{2}\right] \rightarrow \mathrm{POBN}-\mathrm{OH}^{\cdot}+\mathrm{OH}^{\cdot}
$$

The $\mathrm{POBN}-\mathrm{OH}^{\cdot}$ adduct formed has only a lifetime of $23 \mathrm{~s}$ and is thus not stable enough to be detected by ESR under these experimental conditions. The free $\mathrm{OH}^{*}$ can then react with EtOH and POBN to form the POBN-ethoxy adduct observed. Addition of DABCO renders the reaction (1) impossible and, as a direct consequence, $\mathrm{OH}^{\cdot}$ generation by reaction (2). This reaction between ${ }^{1} \mathrm{O}_{2}$ and a spin trap has already been mentioned in the case of $\mathrm{DMPO}^{36}$ but, to the best of our knowledge, a similar reaction between ${ }^{1} \mathrm{O}_{2}$ and POBN is proposed for the first time in the present paper. $\mathrm{OH}^{\cdot}$ and ${ }^{1} \mathrm{O}_{2}$, generated after PPME activation, are well known to be responsible for important cellular damages that could lead to necrosis or apoptosis. ${ }^{37}$ The lack of apoptosis induced by activated-PPME could be a consequence of the small amounts of PPME located in the mitochondria and the high reactivity of ${ }^{1} \mathrm{O}_{2}$ and $\mathrm{OH}^{*}$ (which have a radius of action $<0.02 \mu \mathrm{m}$ in biological systems $^{38}$ ). The relatively short radii of action of those two ROSs probably allows their interaction with organelles in which the fixation of PPME is important (endothelial reticulum, Golgi apparatus and lysosomes). Kessel and co-workers have demonstrated that the subcellular PS-localization, and therefore the nature of the first organelles photodamaged, could be important in the type of cell death induced by PDT. ${ }^{39,40}$ In their case, an absence of mitochondrial PS fixation led to necrosis and delayed or prevented an apoptotic response to PDT. These authors also remarked that this property was not systematic, but depended on the type of cell line-PS association as well as the PS concentration used. ${ }^{39}$ We here advance the hypothesis that photosensitization of HCT-116 cells by PPME also seems to favour necrotic cell death because of the PS subcellular localization. PPME combined with HCT-116 cell line is therefore a potential treatment to eradicate such cells; unfortunately the drug favours the necrotic cell death pathway, causing a risk of inflammation. PPME seems, however, to be more effective at killing cisplatin-resistant ovarian cells, with which $16.71 \%$ of apoptosis has been reached. ${ }^{41}$

\section{Conclusions}

This study has clarified the biodistribution of PPME inside HCT116 cells, with evidence of mitochondrial co-localization of drug. We have also proved the great benefit of encapsulation of PPME in DMPC liposomes. This vectorization of PS improves by a factor of 5 the intracellularization of PPME, for concentrations below the saturation level. This enhancement has been confirmed by fluorescence spectroscopy, by ESR experiments and by flow cytometry analysis. We have also characterized the ROSs engendered by PPME and their implication in the cell death process.

\section{Acknowledgements}

The authors thank S. Ormenese, R. Stephan and the GIGA-Cell Imaging and Flow Cytometry platform for support with flow cytometry.

\section{References}

1 S. Anand, G. Honari, T. Hasan, P. Elson and E. V. Maytin, Low-dose methotrexate enhances aminolevulinate-based photodynamic therapy in skin carcinoma cells in vitro and in vivo, Clin. Cancer Res., 2009, 15, 3333-3343.

2 CM. Moore, D. Pendse and M. Emberton, Photodynamic therapy for prostate cancer - a review of current status and future promise, Medscape, 2009, 6, 18-30.

3 M. Zeisser-Labouèbe, N. Lange, R. Gurny and F. Delie, Hypericinloaded nanoparticles for the photodynamic treatment of ovarian cancer, Int. J. Pharm., 2006, 326, 174-181.

4 M. Bhatti, G. Yahioglu, LR. Milgrom, M. Garcia-Maya, K. A. Chester and M. P. Deonarain, Targeted photodynamic therapy with multiplyloaded recombinant antibody fragments, Int. J. Cancer, 2008, 122(5), 1155-1163. Erratum in: M. Bhatti, G. Yahioglu, LR. Milgrom, M. Garcia-Maya, K. A. Chester and M. P. Deonarain, Int. J. Cancer, 2008, 123(1), 245.

5 A. Kamuhabwa, P. Agostinis, B. Ahmed, W. Landuyt, B. van Cleynenbreugel, H. van Poppel and P. de Witte, Hypericin as a potential phototherapeutic agent in superficial transitional cell carcinoma of the bladder, Photochem. Photobiol. Sci., 2004, 3, 772-780.

6 P. Míkvy, H. Messmann, J. Regula, M. Conio, M. Pauer, C. E. Millson, A. J. MacRobert and S. G. Bown, Photodynamic therapy for gastrointestinal tumors using three photosensitizers - ALA induced PPIX, Photofrin and MTHPC. A pilot study, Neoplasma, 1998, 45, $157-161$.

7 M. Salah, N. Samy and M. Fadel, Methylene blue mediated photodynamic therapy for resistant plaque psoriasis, J. Drugs Dermatol., 2009, 8,42 .

8 G. E. Lang, S. Mennel, G. Spital, J. Wachtlin, B. Jurklies, H. Heimann, B. Damato and C. H. Meyer, Different indications of photodynamic therapy in ophthalmology, Klinische Monatsblätter für Augenheilkunde, 2009, 226(9), 725-739.

9 C. W. So, P. W. Tsang, P. C. Lo, C. J. Seneviratne, L. P. Samaranayake and W. P. Fong, Photodynamic inactivation of Candida albicans by BAM-SiPc, Mycoses, 2010, 53(3), 215-220.

$10 \mathrm{~S}$. Terrell and D. Aires, Treatment of acne vulgaris using blue light photodynamic therapy in an African-American patient, J. Drugs Dermatol., 2009, 8, 669-671.

11 G. R. Reddy, M. S. Bhojani, P. McConville, J. Moody, B. A. Moffat, D. E. Hall, G. Kim, Y. E. Koo, M. J. Woolliscroft and J. V. Sugai, Vascular targeted nanoparticles for imaging and treatment of brain tumors, Clin. Cancer Res., 2006, 12, 6677-6686.

$12 \mathrm{X}$. Sun and W. N. Leung, Photodynamic therapy with pyropheophorbide-a methyl ester in human lung carcinoma cancer cell: efficacy, localization and apoptosis, Photochem. Photobiol., 2002, 75, 644-651.

13 J. Y. Matroule, G. Bonizzi, P. Morlière, N. Paillous, R. Santus, V. Bours and J. Piette, Pyropheophorbide-a methyl ester-mediated photosensitization activates transcription factor NF- $\mathrm{KB}$ through the interleukin-1 receptor-dependent signaling pathway, J. Biol. Chem., 1999, 274, 2988-3000.

14 J. Y. Matroule, C. M. Carthy, D. J. Granville, O. Jolois, D. W. Hunt and J. Piette, Mechanism of colon cancer cell apoptosis mediated by pyropheophorbide-a methylester photosensitization, Oncogene, 2001, 20, 4070-4084.

15 Y. Tian, W. Leung, K. Yue and N. Mak, Cell death induced by MPPaPDT in prostate carcinoma in vitro and in vivo, Biochem. Biophys. Res. Commun., 2006, 348, 413-420.

16 C. S. Xu and A. W. Leung, Photodynamic effects of pyropheophorbidea methyl ester in nasopharyngeal carcinoma cells, Med. Sci. Monit., 2006, 12, 257-262.

17 G. Begum, A. Dube, P. G. Joshi, P. K. Gupta and N. B. Joshi, Chlorin p6 preferentially localizes in endoplasmic reticulum and Golgi apparatus and inhibits $\mathrm{Ca}^{2+}$ release from intracellular store, J. Photochem. Photobiol., B, 2009, 95, 177-184. 
18 Y. Wei, B. Kong, K. Song, X. Qu, Q. Jin and Q. Yang, Involvement of mitochondria-caspase pathway in Hemoporfin-mediated cell death, Photochem. Photobiol., 2007, 83, 1319-1324.

19 E. Alarcón, A. M. Edwards, A. M. Garcia, M. Muñoz, A. Aspée, C. D. Borsarelli and E. A. Lissi, Photophysics and photochemistry of zinc phthalocyanine/bovine serum albumin adducts, Photochem. Photobiol. Sci., 2009, 8(2), 255-263.

20 L. Delanaye, M. A. Bahri, F. Tfibel, M. P. Fontaine-Aupart, A. Mouithys-Mickalad, B. Heine, J. Piette and M. Hoebeke, Physical and chemical properties of pyropheophorbide-a methyl ester in ethanol, phosphate buffer and aqueous dispersion of small unilamellar dimyristoyl-L- $\alpha$-phosphatidylcholine vesicles, Photochem. Photobiol. Sci., 2006, 5, 317-325.

21 M. A. Tran, R. J. Watts and G. P. Robertson, Use of liposomes as drug delivery vehicles for treatment of melanoma, Pigm. Cell Melanoma Res., 2009, 22(4), 388-399.

22 A. Ikeda, Y. Doi, K. Nishiguchi, K. Kitamura, M. Hashizume, J. Kikuchi, K. Yogo, T. Ogawa and T. Takeya, Induction of cell death by photodynamic therapy with water-soluble lipid-membraneincorporated [60]fullerene, Org. Biomol. Chem., 2007, 5(8), 1158-1160.

23 J. Buchholz, B. Kaser-Hotz, T. Khan, C. Rohrer Bley, K. Melzer, R. A. Schwendener, M. Roos and H. Walt, Optimizing photodynamic therapy: in vivo pharmacokinetics of liposomal meta(tetrahydroxyphenyl)chlorin in feline squamous cell carcinoma, Clin. Cancer Res., 2005, 11(20), 7538-7544.

24 J. Kuntsche, I. Freisleben, F. Steiniger and A. Fahr, Temoporfin-loaded liposomes: physicochemical characterization, Eur. J. Pharm. Sci., 2010, 40(4), 305-315.

25 M. J. Hope, M. Bally, G. Webb and P. R. Cullis, Production of large unilamellar vesicles by a rapid extrusion procedure. Characterization of size distribution, trapped volume and ability to maintain a membrane potential, Biochim. Biophys. Acta, Biomembr., 1985, 812, 55-65.

26 L. D. Mayer, M. J. Hope and P. R. Cullis, Vesicles of variable sizes produced by a rapid extrusion procedure, Biochim. Biophys. Acta, Biomembr., 1986, 858, 161-168.

27 F. Olson, C. A. Hunt, F. C. Szoka, W. J. Vail and D. Papahadjopoulos, Preparation of liposomes of defined size distribution by extrusion through polycarbonate membranes, Biochim. Biophys. Acta, Biomembr., 1979, 557, 9-23.

28 S. Bolte and F. P. Cordelieres, A guided tour into subcellular colocalization analysis in light microscopy, J. Microsc., 2006, 224, 213-232.
29 S. V. Costes, D. Daelemans, E. H. Cho, Z. Dobbin, G. Pavlakis and S. Lockett, Automatic and quantitative measurement of protein-protein colocalization in live cells, Biophys. J., 2004, 86, 3993-4003.

30 L. S. Alexander-North, J. A. North, K. P. Kiminyo, G. R. Buettner and A. A. Spector, Polyunsaturated fatty acids increase lipid radical formation induced by oxidant stress in endothelial cells, J. Lipid Res., 1994, 35(10), 1773-1785.

31 E. Finkelstein, G. Rosen and E. Rauckman, Spin trapping of superoxide, Mol. Pharm., 1979, 16, 676-685.

32 S. Pou, C. L. Ramos, T. Gladwell, E. Renks, M. Centra, D. Young, M. S. Cohen and G. M. Rosen, Does peroxynitrite generate hydroxyl radical?, Anal. Biochem., 1994, 217, 76-78.

33 Y. N. Konan and R. G. Allémann, State of the art in the delivery of photosensitizers for photodynamic therapy, J. Photochem. Photobiol. $B, 2002,66,89-106$.

$34 \mathrm{H}$. Hillaireau and P. Couvreur, Nanocarriers' entry into the cell: relevance to drug delivery, Cell. Mol. Life Sci., 2009, 66, 28732896.

35 K. Kuida, T. F. Haydar, C. Y. Kuan, Y. Gu, C. Taya, H. Karasuyama, M. S. Su, P. Rakic and R. A. Flavell, Reduced apoptosis and cytochrome c-mediated caspase activation in mice lacking caspase 9, Cell, 1998, 94, 325-337.

36 M. Hoebeke, H. J. Schuitmaker, L. E. Jannink, T. M. Dubbelman, A. Jakobs and A. Van de Vorst, Electron spin resonance evidence of the generation of superoxide anion, hydroxyl radical and singlet oxygen during the photohemolysis of human erythrocytes with bacteriochlorin a, Photochem. Photobiol., 1997, 66(4), 502-508.

37 B. Halliwell and J. M. C. Gutteridge, in Free radicals in Biology and Medecine, Oxford University Press, Oxford, UK, 3rd edn, 1999.

38 E. Buytaert, M. Dewaele and P. Agostinis, Molecular effectors of multiple cell death pathways initiated by photodynamic therapy, Biochim. Biophys. Acta, 2007, 1776, 86-107.

39 D. Kessel and Y. Luo, Mitochondrial photodamage and PDT-induced apoptosis, J. Photochem. Photobiol., B, 1998, 42, 89-95.

40 D. Kessel, Y. Luo, Y. Deng and C. K. Chang, The role of subcellular localization in initiation of apoptosis by photodynamic therapy, Photochem. Photobiol., 1997, 65, 422-426.

41 Y. Tan, C. S. Xu, X. S. Xia, H. P. Yu, D. Q. Bai, Y. He and A. W. Leung, Photodynamic action of LED-activated pyropheophorbide- $\alpha$ methyl ester in cisplatin-resistant human ovarian carcinoma cells, Laser Phys. Lett., 2009, 6, 321-327. 